Pilot studies guide the development of research plans to ensure that the methods and ideas which are being proposed will work in practice (Kim, 2010). This pilot study was undertaken to explore the feasibility of a proposed Doctorate study designed to answer the research question: 'What is the family experience of bereavement when a family member has dementia and what might this mean for the provision of support?' using constructivist grounded theory (Charmaz, 2014).

The pilot explored the family bereavement experience from the perspective of one family member and focused on the use of unstructured interviews and research diaries as tools for quality data collection, and a process of data analysis involving process coding and memo writing (Saldaña, 2016).

Tentative theoretical understanding of the data emerged including:

- Experiencing a spiritual dimension

- Being a bereaved carer

- Family functioning

- Relationship between bereavement and dementia.

These early findings will be integrated into the main study through the constant comparison process consistent with grounded theory methodology (Charmaz, 2014; Urquhart, 2013).

Carrying out and reflecting on the pilot highlighted several issues including the researchers' role within the interview and data analysis process, the design and use of a diary for data collection and the ethical dilemma of involving or excluding people with dementia within the study. Learning from this pilot will help shape future study plans.

\section{Communication, voices and stories}

\section{P-14 EXPLORATION OF SUPPORT WORKERS' AND VOLUNTEERS' INITIATION OF QUALITY OF LIFE CONVERSATIONS}

${ }^{1}$ Sharan Watson, ${ }^{2}$ Alison Hembrow. ${ }^{1}$ University of Derby, Derby, UK; ${ }^{2}$ Treetops Hospice Care, Derby, UK

\subsection{6/bmjspcare-2018-hospiceabs.39}

Background Through being a pilot site for NHS England exploring Personal Health Budgets (PHBs) in end of life care, Treetops Hospice Care became aware that there needed to be more focus on conversations around what was important to patients. There appeared to be barriers around developing these conversations, possible reasons were lack of healthcare professionals' time and clarity as to whose role it was. Resulting from this highlighted need, Treetops Hospice Care and the University of Derby are undertaking a research project to explore the outcomes of developing support workers and volunteers in initiating conversations around quality of life. Policy drivers have acknowledged that there is a much greater need and demand for person-centred care than professionals in health and social care can meet (Health Education England, Skills for Health \& Skills for Care, 2017). This follows the principles of a 'public health' approach to end of life care, and that a 'de-professionalisation' of supportive care, involving volunteers and support staff engaging in these conversations, could result in being able to include more individuals (Abel \& Kellehear, 2016).
Aims Exploring participants' involvement and perceptions of patient-centred conversations to improve end of life care experiences.

Methods/conclusion Qualitative interviewing will be undertaken before and after a new educational training approach is delivered, along with a pre and post Likert evaluation tool capturing self-perceived confidence and competence. The new approach will focus around the mnemonic L.I.S.T.E.N (developed by Treetops Hospice Care) supporting these conversations around preferences and wishes, within a person-centred approach.

We anticipate that with the appropriate support and training, unregistered healthcare professionals/volunteers feel more enabled in having these conversations, empowering them to offer more timely conversation opportunities with palliative care patients. Resulting outcomes will inform future training.

\section{P-15 BESPOKE HOSPITAL DISCHARGE SUMMARY GUIDES COMMUNICATION OF PATIENT PREFERENCES AT END OF LIFE}

Jean Potter, Liz Bunker, Eleni Baldwin, Marc Magee. The Hillingdon Hospital NHS Foundation Trust, London, UK

10.1136/bmjspcare-2018-hospiceabs.40

Introduction Identification of patients approaching end of life, and advance care planning (ACP) with them, can improve patient outcomes and reduce hospital deaths.

Methods The essential items that should be included in any initial ACP discussions were agreed by a working group (COTE doctor, palliative care doctor and nurse, GP and Patient in Partnership Group). Some items were mandatory and some dynamic. Items included:

1. Dropdown menu: prognostication (based on SPICT)

2. Yes/No: elements of discussions had, e.g. regarding prognostication, resuscitation status during stay, Preferred Place of Care, Preferred Place of Death

3. Free text: e.g. ceilings of care.

4. These items were added to the usual hospital discharge summary template (EPRO) by the Trust's IT department.

Patients were identified as appropriate for invitation to ACP discussion by using a modified Surprise Question (found to have $61 \%$ sensitivity and $88 \%$ specificity in this group), which was used as standard during COTE MDT discussions.

Following a short training session to COTE team, at discharge the modified discharge summary was used for appropriate patients.

COTE discharge summaries were surveyed using a standardised proforma to quantify recording of ACP preferences ( $n=40$ at baseline and $n=2010$ weeks after new template introduced).

Results The new ACP template improves frequency of documentation in discharge summaries from baseline of: resuscitation status $(10 \%$ to $100 \%)$ information regarding prognostication $(0 \%$ to $100 \%)$ and any mention of patient preferences for care (8\%-100\%).

Conclusion Specific items added to the hospital discharge summary at no extra cost improves ACP information sharing from secondary to primary care. Staff fed back that the ACP discharge summary fields were easy to use. The teams continue to engage with the process as they see improved cross 
boundary communication, and it is useful upon any readmission as discharge summaries are more readily accessed than the paper notes.

\section{P-16 SIMPLE, ACCURATE IDENTIFICATION OF APPROPRIATE HOSPITAL INPATIENTS FOR ADVANCE CARE PLANNING BY COTE}

Jean Potter, Roberta Jordan, Catherine Pye, Yolande Saunders. The Hillingdon Hospital NHS Foundation Trust, London, UK

10.1136/bmjspcare-2018-hospiceabs.41

Background Identification of patients approaching the end of life and advance care planning (ACP) with them can improve patient outcomes and reduce hospital deaths. But this aspect of care often receives little attention in the hospital inpatient setting.

Aims (i) To estimate the proportion of a sample of COTE hospital inpatients that are identified, by their own team, as likely to be in the last $6-12 / 12$ of life; (ii) To describe these patients in terms of: likelihood of death in 6-12/12 using Gold Standards Framework Prognostic Indicator Guidance (GSF PIG); clinical complexity (using the ACE27 comorbidity score); content of any advance care plans; (iii) To ascertain the number of patients from this sample who died in the subsequent $6 / 12$.

Methods COTE Consultant led MDTs prognosticated 57 inpatients under their care during one week in March 2016, using two screening questions. All patients thought to have an at least moderate expectation of death (based on a modified Surprise Question: 'Would you be surprised if this patient were still alive in the next 6-12/12?') had their notes reviewed using a standardised proforma. Six months later the number of deaths was ascertained using hospital and community databases.

Results $70 \%$ patients were considered likely to be in the last $6-12 / 12$ of life. $98 \%$ had this prognostication confirmed with GSF PIG. 68\% patients had complex care needs, but few had advance care plans recorded. Over one third died within six months. The modified Surprise Question correctly identified patients who went on to die in the following six months with a sensitivity $61 \%$ and specificity $88 \%$.

Discussion Many COTE inpatients are in the last 6-12/12 of life. One question identifies $>60 \%$ with high specificity, and this is now an integral part of the weekly COTE MDT discussion, and will prompt ACP discussions with patients and families.

\section{P-17 ADVANCE CARE PLANNING IN DEMENTIA - TRAINING NEEDS ANALYSIS FOR EFFECTIVE FACILITATION}

Karen Harrison Dening, Caroline Scates. Dementia UK, London, UK

10.1136/bmjspcare-2018-hospiceabs.42

Advance care planning (ACP) is a voluntary process of discussion and review to help an individual who has capacity to anticipate how their condition may affect them in the future. Dementia is a progressive and life limiting illness (Sampson \& Harrison Dening, 2013), where the mental capacity to make decisions can be lost fairly early on in the illness. There is a small, growing literature on ACP and its application in dementia in the UK highlighting that its uptake is small and patchy due to several factors. People with a life-limiting illness, especially dementia, are not routinely consulted about their wishes and preferences for future care. There are several potential barriers that may contribute to this in the general population, such as, procrastination, or waiting to do it later, dependence on family for decision making. However, in dementia, there are several additional barriers to initiating ACP one of which is a reluctance on the part of professionals to undertake such difficult initial conversations. This is in part due to the perception that they lack the knowledge, skills and experience to do so (Harrison Dening, Jones \& Sampson, 2011).

Admiral Nurses (AN) are expert practitioners in the case management of families affected by dementia. A growing aspect of their role is in supporting ACP. However, as more and more are recruited Dementia UK wanted to ensure there was a consistency across the workforce in their ability to facilitate the effective initiation and development of ACP. This paper discusses a UK-wide training needs analysis of ANs undertaken during master classes on palliative and end of life care in dementia. It will discuss results and the development of an educational intervention to equip ANs in this activity.

\section{P-18 ANTICIPATORY CARE PLANNING ON AN ACUTE GASTROENTEROLOGY WARD}

Karim El-Shakankery, Daisy Riddle, Lucy Pain. Queen's Hospital, Romford, London, UK

\subsection{6/bmjspcare-2018-hospiceabs.43}

Background With a rapidly ageing population and advances in medicine, hospital inpatients are increasingly frail with complex needs, and frequently die in hospital. Advance Care Planning (ACP) is a valuable process supporting clinicians to provide patients with the right care, at the right time, in the right place.

The Gold Standards Framework (GSF) is a structured approach to ACP for patients likely to be in their last year of life. In our Trust geriatric wards have been using GSF for several years, however acute medical wards have less experience of ACP, despite many of their inpatients being in their last year of life due to both malignant and non-malignant conditions.

Aims To assess the quality and quantity of ACP on a Gastroenterology ward at Queen's Hospital, Romford before and after implementation of GSF. We also aimed to explore:

- The proportion of inpatients dying within one year

- Significant diagnoses

- Impact of ACP on length of stay, number of readmissions and place of death.

Methods Retrospective review: We identified all admissions to the ward over June 2017. All patients dying within one year were selected; data was extracted from medical notes. Data included diagnosis, length of stay, number of subsequent admissions, preferred place of care, DNAR status, and use of any formal ACP documentation.

Prospective audit: In June 2018 we implemented GSF to the ward. All patients identified were offered ACP. Comparable data was collected prospectively.

Results In June 2017, 106 patients were admitted to the ward; of these 27 have died to date. We continue to analyse 